\title{
Camera-based micro interferometer for applications in distance sensing
}

\author{
Matthias Will'; Andreas Winzer', Martin Schädel' ${ }^{2}$,Jan Freitag², Olaf Brodersen² \\ ${ }^{1}$ Hochschule Darmstadt, Fachbereich Mathematik und Naturwissenschaften, Schöfferstrasse 3, \\ D-64295 Darmstadt, Germany, \\ matthias.will@h-da.de \\ ${ }^{2}$ CiS Forschungsinstitut für Mikrosensorik GmbH Konrad-Zuse-Str. 14 D-99099 Erfurt, Germany
}

\begin{abstract}
:
Interference of light provides a high precision, non-contact and fast method for measurement method for distances. However, capacitive, resistive or inductive methods dominate in the field of compact sensors. The reason is, that the interferometric system has to be precise adjusted and needs a high mechanical stability. We developed a new concept for a very small interferometric sensing setup. Therefore we combine a miniaturized laser unit, a low cost pixel detector and machine vision routines to realize a demonstrator for a Michelson type micro interferometer. Furthermore we develop a concept which allows the automatable production without individual adjustment. We demonstrate a low cost sensor smaller $1 \mathrm{~cm}^{3}$ including all electronics and demonstrate distance sensing up to $30 \mathrm{~cm}$ and resolution in $\mathrm{nm}$ range.
\end{abstract}

Key words: distance sensing, VCSEL, micro interferometer, adjustment free

\section{Introduction}

The interference of light provides a possibility for a precise distance measurement. The comparison with a reference beam allows to measure a relative length in wavelength units via simple counting of bright-dark transitions. Techniques such as interpolation, wavelength or phase modulations allow absolute measurements and resolutions in the subnanometer range.

The use of monochromatic lasers with high coherence length and low divergence makes it possible to realize the most accurate length measurements in research and is also established in the industrial fields like wafer steppers, calibration tools, vibrometry and other. Outstanding precision and measurement ranges by a contactless method are not achievable by any other techniques. [1]

Nevertheless, the major role in applications are inductive, capacitive or optically read encoders. The causes for this are, that the different methods allows the realization of optimally adapted systems in relation to technical requirements and device costs. Especially systems can be realized as a small sensor. The laser interferometer, compared with these, is usually a table-top device whose costs are in the range of several thousand euros.
The reason for this are on the one hand, the requirements of the laser itself. Here the inefficient and expensive gas laser is the most common source. Add to that, we have the high stability requirements of an individual adjustable mechanical setup.

The optical microsystem technology shows a lot of progress in the last decades. Driven by multimedia applications such as DVD pickup systems, mobile phone cameras, LED and technology, new ways are possible.

The aim of the work was to investigate how an interferometric micro sensor, with potential of low production costs, can be realized.

\section{Target parameter and applications}

In the last 20 years there was intensive investigations for building micro spectrometers. Despite intensive efforts, there are many different types and applications but not a really low cost device and a mass production. The problem was often the discrepancy between demands of applications and performance loss due to miniaturization. We can learn from this that the real performance of a miniaturized system are hard to predict and only with proof of concept studies reliable. Based on this experiences, we evaluate the specifications and cost areas with highes success potential. 
We identify two application fields from interest. First in the application field of capacitive sensors. The accuracy of these can't be surpassed, but the interferometric principle allows for a larger measuring range, a constant resolution, smaller measuring fields and a larger working distance. (Note, the accuracy of capacitive sensors decreases with increasing distance and smaller size of electrodes) But capacitive sensors are well established, achieve very high resolution, stability and low cost. Therefore, we focused on a second region in the transition from inductive sensing to the optical encoders. The limit of the typical digital dial gauge which is uses in a high numbers is about $1 \mu \mathrm{m}$ with a measuring range about $1 \mathrm{~cm}$ and system costs of a few $100 €$. The optical encoder based system are usual 10 times more expensive.

As a consequence we define the following market-relevant target parameters for the system we have to realize:

- measuring range $1 . .10 \mathrm{~cm}$ with resolution less than $100 \mathrm{~nm}$ (better than similar inductive systems)

- measuring range $0,1 \ldots 1 \mathrm{~mm}$ and resolution less than $1 \mathrm{~nm}$ (similar capacitive systems)

- $\quad$ size the complete system $<1 \mathrm{~cm}^{3}$

- Material cost should be below $100 €$ manufacture time less than $1 \mathrm{~h}$

\section{The sensor concept}

Conventional interferometers on the market are mainly based on a Michelson interferometer setup. Additional a dynamic phase shift of the reference beam is necessary to avoid influence of Light intensity and detect direction of movement. This can be realized by an oscillation of the reference mirror, but we want to avoid moving parts in our micro system. Another concept which is used are generation of interference fringes and the detection of comb like photodiodes. These type of interferometers are very fast because they are not limited on the oscillation frequency of the reference phase. In conclusion we have to integrate a laser, collimation optics, beam splitter, reference mirror and photo detector inside $1 \mathrm{~cm}^{3}$. For fabrication demands we have to use micro system technologies, which means components should be able to use in to pick and place automation and everything we want to fix by gluing. The passive assembled interferometer system should be working with these typical tolerances. In consequence we found two conditions. First, the highest position precision is necessary between VCSEL chip and micro lenses. So we need a preassembled and tested monolithic light source. The expected variations of interference pattern without adjustment requires an intelligent detector.

\section{The Light emitter}

Due to the size we want to have in any case only laser diodes can be used. Furthermore we need a coherence length in $\mathrm{cm}$ range and narrow bandwidth. In conclusion only singlemode Laser diodes can meet this requirements. We decide to use a single-mode VCSEL. These were use in large volume telecommunication applications. Therefore the cost are relatively low. Most of the singe mode VCSEL are available in wavelength range about $850 \mathrm{~nm}$. But due to eye-safety regulations and for better visibility of measurement beam we select a Laser diode of $670 \mathrm{~nm}$ and $0,5 \mathrm{~mW}$ optical power (Optowell SS67-4U). For distance calculations we need a stable wavelength. It was shown that the wavelength of this laser diode can be stabilizes in pico meter range by using peltier elements already available in a $5 \mathrm{~mm}$ TO housing. But the size of this housing doesn't fit to our system concept. So we abstain from temperate stabilization for the demonstrator and focus on the collimation optic and high integrates packaging.

\section{The monolithic laser element [2]}

The used lightsourse element was developed and fabricated with technology elements of the $\mathrm{CiS} \mathrm{GmbH}$. The footprint of a single laser unit was $700 \times 700 \mu \mathrm{m}^{2}$ and a length of $1.5 \mathrm{~mm}$.
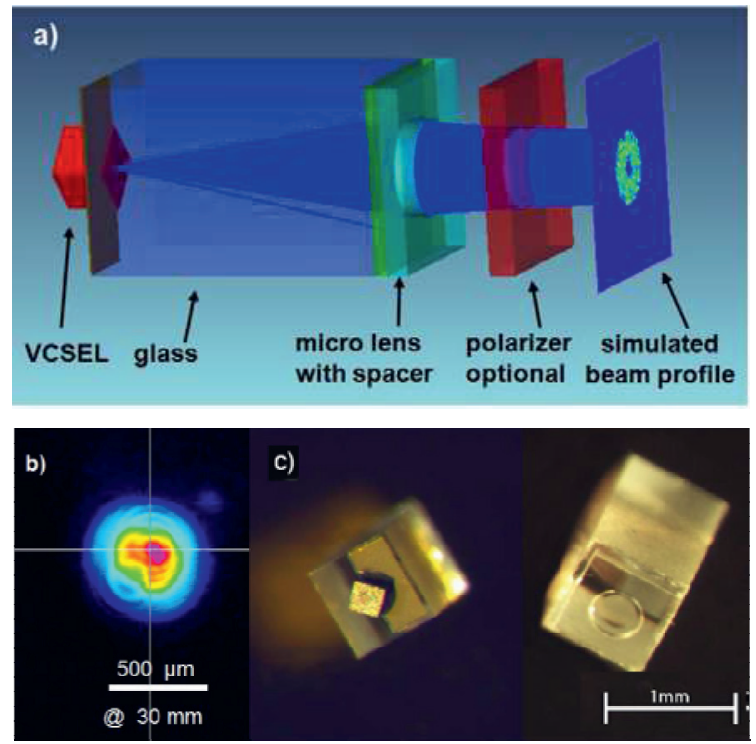

Fig.1. a) Design and simulated beam profile of the laser illumination unit. b) Measured beam profile at a distance of $30 \mathrm{~mm}$ from the lens. The $1 / \mathrm{e}^{2}$ beam diameter was determined with $310 \mu \mathrm{m} . c)$

Microscopic photography of the laser illumination unit from the laser diode side and the lens side. 
The light source shows a well collimated beam with $0.3 \mathrm{~mm}$ diameter at light source entrance. The technological process chain combines a batch based process with the ability to use offthe-shelf laser diodes. The substrate sizes range from 100 to up to $200 \mathrm{~mm}$ with 3500 and 15000 units per wafer, respectively. The excellent optical performance and cost potential is perfect for application in the interferometric micro sensor.

\section{The detector element}

The principle of detection is the detection of position of an interference pattern. In ideal configuration parallel and perpendicular stripes. One can see the tolerances for position of mirrors, beam splitter, laser etc. is definitely below reachable by passive mounting of $\mathrm{mm}$ size components and gluing. So we need a flexible matrix detector and an intelligent evaluation routine. For our demonstrator we use a standard $640 \times 480$ pixel CMOS detector with active area of $1.7 \mathrm{~mm}^{2}$. The chip and conversion electronic to USB standard was mounted on a small PCB. Whole size of the detector unit is $19 \mathrm{~mm} \times 5 \mathrm{~mm} \times 2 \mathrm{~mm}$.

\section{The sensor setup and assembly}

To assemble are 4 components, the PCB with CMOS detector, beam splitter cube, reference mirror and light source. We use a manual fine placer. For connection we dispense UV hardening glue on the optical surfaces. All surfaces are in the same size, so we have a strong self-adjustment effect. After realize the top component the surface tension of the glue centers the positions. There is no need for any precise adjustment. To adapt the size of the light source footprint we use a $3 \times 3$ array with only one emitter in the middle element.

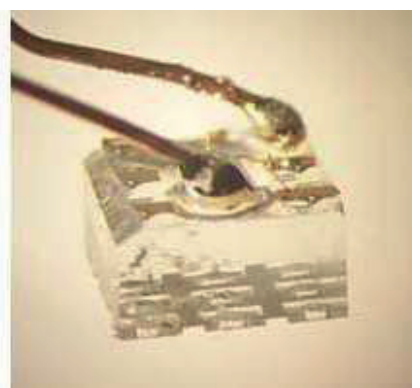

Fig.2. The $3 \times 3$ lens array. Additional pads used for soldering of connection wires. VCSEL contact to solder pads by wire bonding.

To bring the reference mirror in tilted position, we use a two-step hardening and additional contact with the fine placer tool. The angle can be defined by the amount of the glue. After soldering the two connection wires of the lightsource the system was stabilized by a glob top shed.

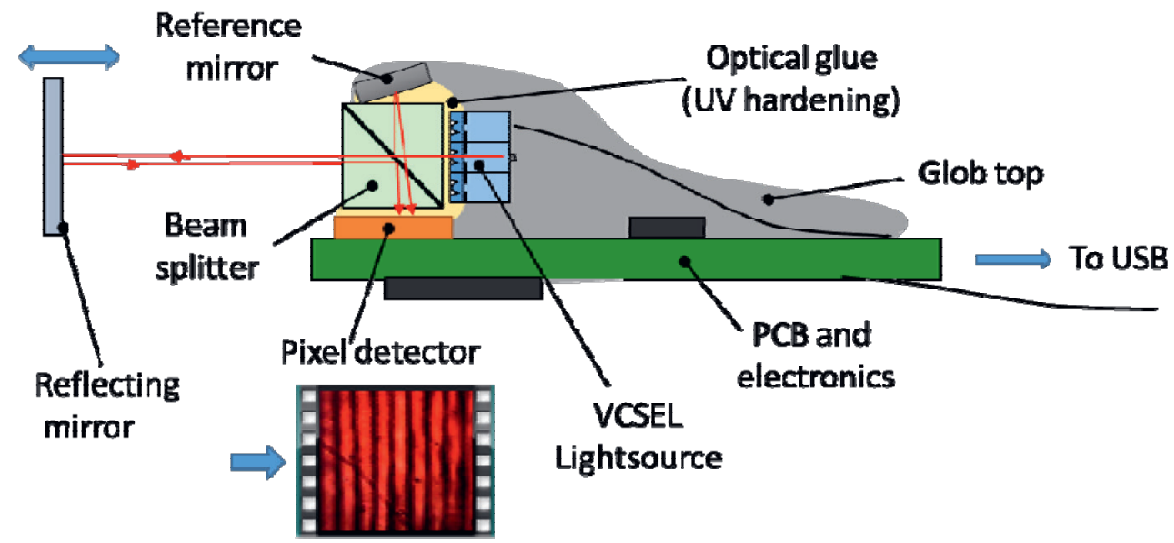

Fig.3. The sensor setup
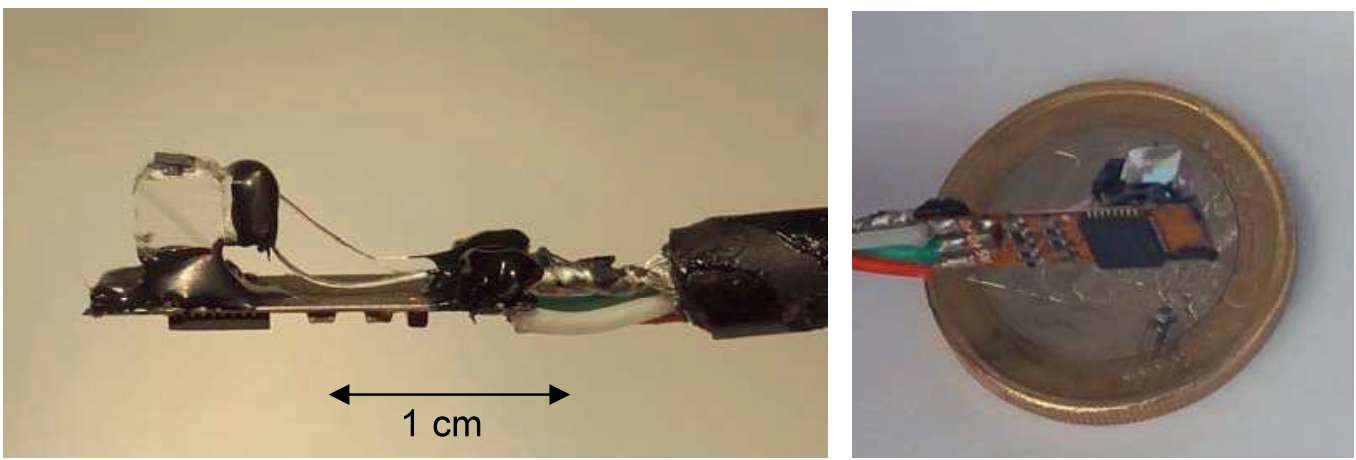

Fig.4. Foto of the interferometric distance sensor 


\section{Sensor evaluation}

To elevate the sensor we use piezo stage with $20 \mu \mathrm{m}$ travel range for defines moving of the reflection mirror. The angle of the mirror can be adjusted to reflect the beam back to the prism entrance. Furthermore the piezo stage was mounted on a rail system to test distances in $\mathrm{cm}$ range. Typical interference pattern depending on adjustment are shown in the pictures. One can see, it is impossible to detect the pattern with a fixed detector. But it is not much difficult to detect period and phase by image processing in all cases.
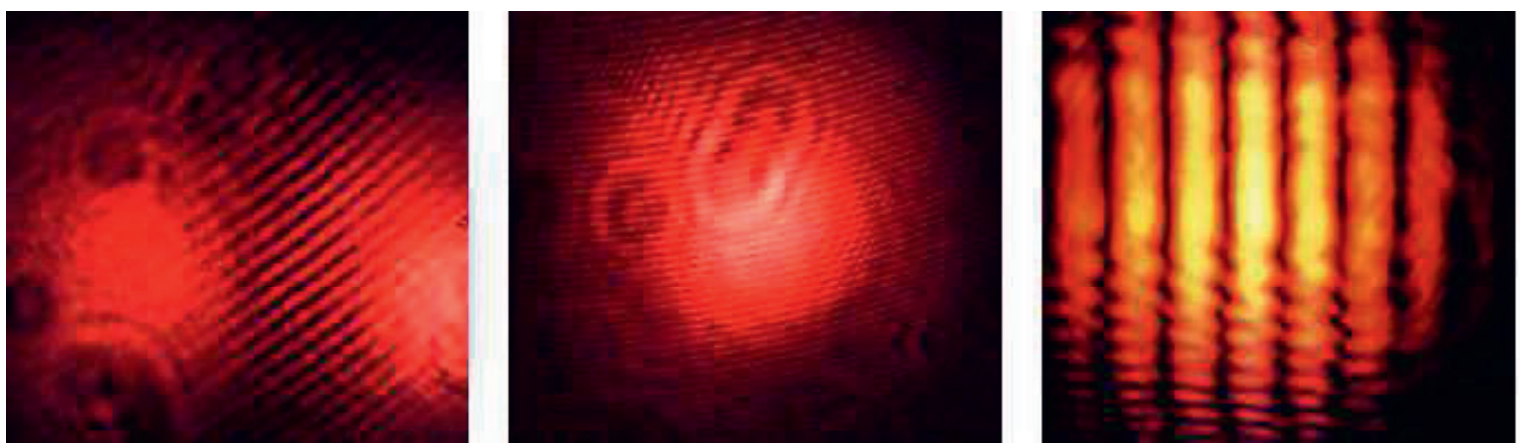

Fig.5. Typical intensity distribution on the CMOS detector. Left: Reference mirror close to sensor, reflected Beam without direct overlap Middle: Mirror in $25 \mathrm{~cm}$, reflected beam is now larger than CMOS-chip and generate low contrast. Right: Well-adjusted system.

\section{The image processing routine}

To extract the position signal we use a Fast Fourier Transform Algorithm for image rotating and filtering. We extracting the period and phase values from a back transformed image. A phase change of one period corresponds with a distance change of half of the laser wavelength $(670 \mathrm{~nm})$. The principle procedure is the following:

1. Define an range of interest based on intensity histogram

2. FFT transformation and extracting rotation angle

3. Image rotation to perpendicular fringes

4. Frequency filtering (narrow bandwidth around strip period)

5. Back transformation to intensity image

6. Average of all coulombs (gives one row with an sinus signal)

7. Sinus fitting and calculation of phase and period
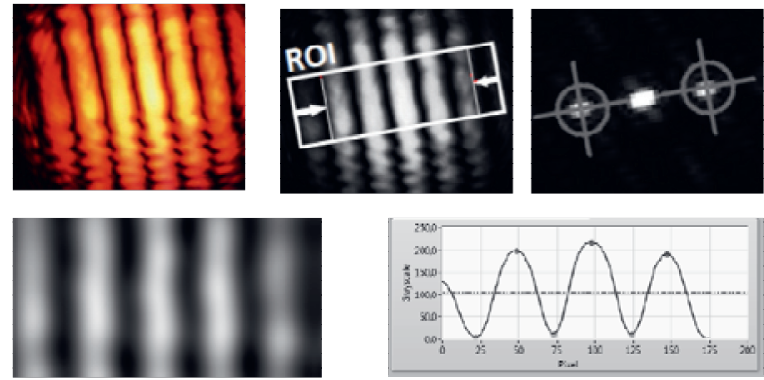

Fig.6. The image processing. Top, from left to right: Original image, ROI, FFT of ROI (Position of the two peaks are characteristic for rotation angle and period), bottom: filtered and back transformed image, right the column average signal.

To find the period signal direct in the FFT image is faster in principle, however with back transformation and average we observe a higher precision and algorithm stability.

\section{Results}

The travel range is limited by coherence length of the laser diode. To test the capabilities of the system we use a well-adjusted reflection mirror and evaluate the contrast of the image (after frequency filtering and rotation). We observe an evaluable contrast up to $30 \mathrm{~cm}$. 

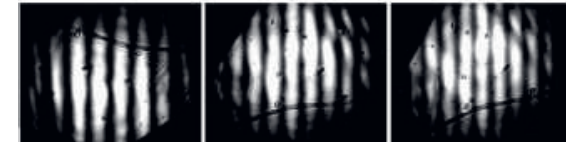

$\Delta d=20 \mathrm{~mm}$

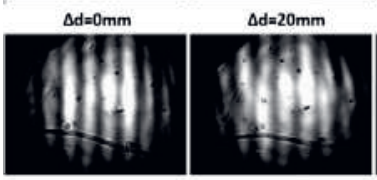

$\Delta d=40 \mathrm{~mm}$

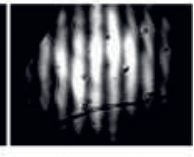

$\Delta \mathrm{d}=60 \mathrm{~mm}$
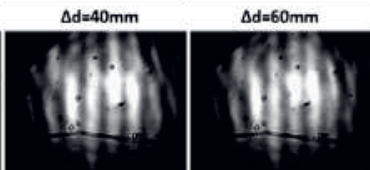

$\Delta d=80 \mathrm{~mm}$ $\Delta d=100 \mathrm{~mm}$ $\Delta d=120 \mathrm{~mm}$

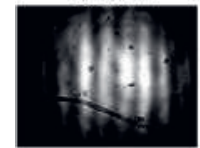

$\Delta d=160 \mathrm{~mm}$

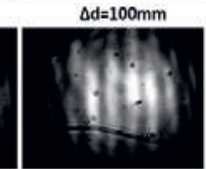

$\Delta d=250 \mathrm{~mm}$

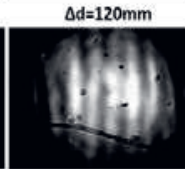

$\Delta d=300 \mathrm{~mm}$

$\Delta \mathrm{d}=140 \mathrm{~mm}$

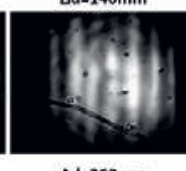

$\Delta d=360 \mathrm{~mm}$

Fig.7.Inerference pattern at different positions of the reflecting mirror.

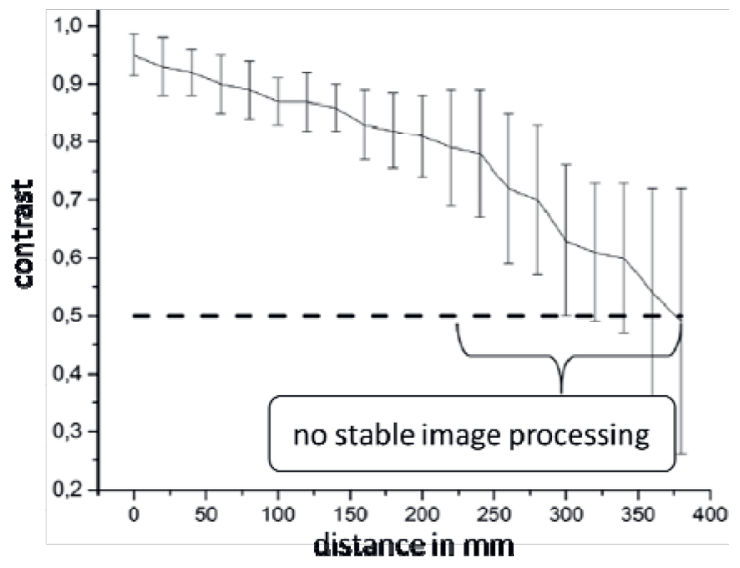

Fig.8.Evaluation of coherence length of the laser diode

But it was not possible to get this without readjusting the mirror. Our testing scheme for is shown in following. We use a piezo actor controlled by a linear voltage ramp. The travel range was always $19.7 \mu \mathrm{m}$. In consequence we have 50 distance points per second. The slop and peak level was used to identify the working range of the system.

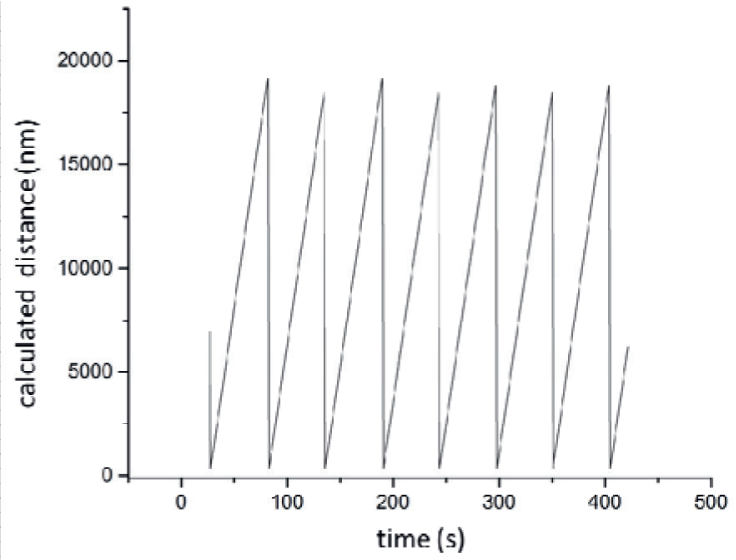

Fig.9.Evaluation of distance measurement

If we optimise to the highest contrast at on position, we observe about $\pm 5 \mathrm{~cm}$ working distance around.

\section{Summary and outlook}

We realise an interferometry distance sensor smaller than $1 \mathrm{~cm}^{3}$ volume, a manufacture, concept without active adjustment, realistic device costs for real world applications, Nano meter resolution, sub-micron accuracy at measuring ranges more than $10 \mathrm{~cm}$. Key elements are pre-adjusted VCSEL micro light source, CMOS pixel detector and intelligent machine vision signal processing.

Most inhibition problem to solve is the max. detectable moving velocity. In time below $0.1 \mathrm{~mm} / \mathrm{s}$ ! To overcome these we will use the combination of two concepts:

First the integration of additional light sources with different wavelengths. Result is a much higher area of uniqueness (in time only halve of wavelength). System in ready for these because the Lightsorce element can hold 9 VCSEL in $2.5 \times 2.5 \mathrm{~mm}^{2}$.

Second the increase the camera frame rate from $50 \mathrm{fps}$ to $400 \mathrm{fps}$. With both we expect max. moving speed about $1 \mathrm{~cm} / \mathrm{s}$, what is sufficient for a large number of applications.

Furthermore we have to stabilize or monitor the wavelength of the light source and evaluate temperature range $\left(10^{\circ} \mathrm{C} \ldots 50^{\circ} \mathrm{C}\right.$ expected) and long-time stability. Detailed investigations are under progress.

\section{References}

[1] Sergio Martellucci, Arthur N. Chester, Anna Grazia Mignani; Optical Sensors and

Microsystems; ISBN: 978-0-306-46380-8 (Print) 978-0-306-47099-8 (Online) VI.

[2] A. T. Winzer, J. Freitag, P. Dannberg, M. Hintz, M. Schädel, V. Sandhya and H.-J. Freitag, A miniaturized laser illumination module, Proc. SPIE 9626, Optical Systems Design 2015: Optical Design and Engineering

[3] Vixar single-mode Laserdiode with TEC Cooler, Datasheet, www.vixarinc.com 